\title{
Apoplejía de adenoma hipofisario no funcionante tras anestesia espinal. A propósito de dos casos
}

\author{
J. González-Tortosa; M Poza-Poza y A. Ruiz-Espejo-Vilar
}

Servicio de Neurocirugía. Hospital Universitario Virgen de la Arrixaca. Murcia.

\section{Resumen}

Se presentan dos casos clínicos de apoplejía hipofisaria tras anestesia espinal, ambos con cefalea y una parálisis del III par craneal. Uno de ellos con alteración visual adicional e hiponatremia. Se revisa la bibliografía en busca de las claves para un diagnóstico precoz y se propone la hipotensión arterial como mecanismo patogénico de la apoplejía.

PALABRAS CLAVE: Adenoma de hipófisis. Apoplejía hipofisaria. Anestesia espinal.

Pituitary adenoma apoplexy after spinal anaesthesia. Report of two cases and review of the literature

\section{Summary}

Two cases of pituitary apoplexy occurring after spinal anaesthesia are described. Both presented with headache and III par palsy, one of them with additional visual disturbance and hyponatremia. A bibliographical review has been done, looking for early diagnostic keys. We propose the arterial hypotension as a possible pathogenetic mechanism of pituitary adenoma apoplexy.

KEYWORDS: Pituitary adenoma. Pituitary apoplexy. Spinal anesthesia.

\section{Introducción}

Los adenomas de hipófisis no funcionantes pueden, a veces, alcanzar un tamaño considerable de forma silente o con escasos síntomas, no siendo raro que se descubran al practicar una prueba de imagen con otro motivo. Tampoco es infrecuente que, la primera manifestación clínica que produzcan, sea una cefalea de inicio brusco, dentro del marco de la clásica apoplejía hipofisaria ${ }^{4,8,10,36,64}$ en la que

Recibido: 6-01-08. Aceptado: 9-02-09 suelen existir náuseas, vértigo, meningismo y/o un nivel alterado de consciencia. Con frecuencia se encuentra oftalmoplejía, deterioro de la agudeza visual y reducción de los campos visuales. En general es un cuadro clínico llamativo, aunque puede cursar de forma más discreta e incluso, ser silente desde el punto de vista clínico ${ }^{64}$. El diagnóstico se confirma con la presencia de lesiones sellares en la TAC o RM que pueden mostrar características de infarto o hemorragia.

Una de las complicaciones habituales de la anestesia espinal, es la cefalea post-punción dural ${ }^{21} \mathrm{y}$ en este punto, puede enmascararse la apoplejía de un adenoma de hipófisis, sometiendo al paciente a un manejo inadecuado y, lo que es más trascendente, retrasándose el diagnóstico y el tratamiento de la verdadera etiología de la cefalea.

Sólo hemos encontrado, en la literatura médica, un caso de apoplejía hipofisaria tras anestesia espinal ${ }^{33}$. Con estos dos pacientes adicionales que presentamos, queremos subrayar que la apoplejía hipofisaria debe de tenerse en cuenta para el diagnóstico diferencial de la cefalea tras una anestesia espinal.

\section{Casos clínicos}

\section{Caso 1}

Varón de 61 años, sin antecedentes de patología cardiaca, diagnosticado de hipercolesterinemia e hipertensión arterial de muchos años de evolución, controlada con medicación. El paciente venía padeciendo de impotencia sexual desde hace 1 año y de una cefalea moderada varias semanas antes de ser sometido, en otro hospital, a una operación de menisco de rodilla, bajo anestesia raquídea con $10 \mathrm{mg}$ de bupivacaina $0,5 \%$. Durante la operación la presión arterial decreció desde 120 a 80 mm Hg, permaneciendo en ese nivel durante tres horas y, el pulso, en 30-45 $\mathrm{s} / \mathrm{m}$. En el inmediato postoperatorio el enfermo sufrió una agudización grave de la cefalea, junto con un cuadro confusional leve y fiebre de $39^{\circ}$. La cefalea era generalizada, opresiva y no relacionada con la posición ortostática. Cuarenta y ocho horas más tarde el paciente presenta pérdida 


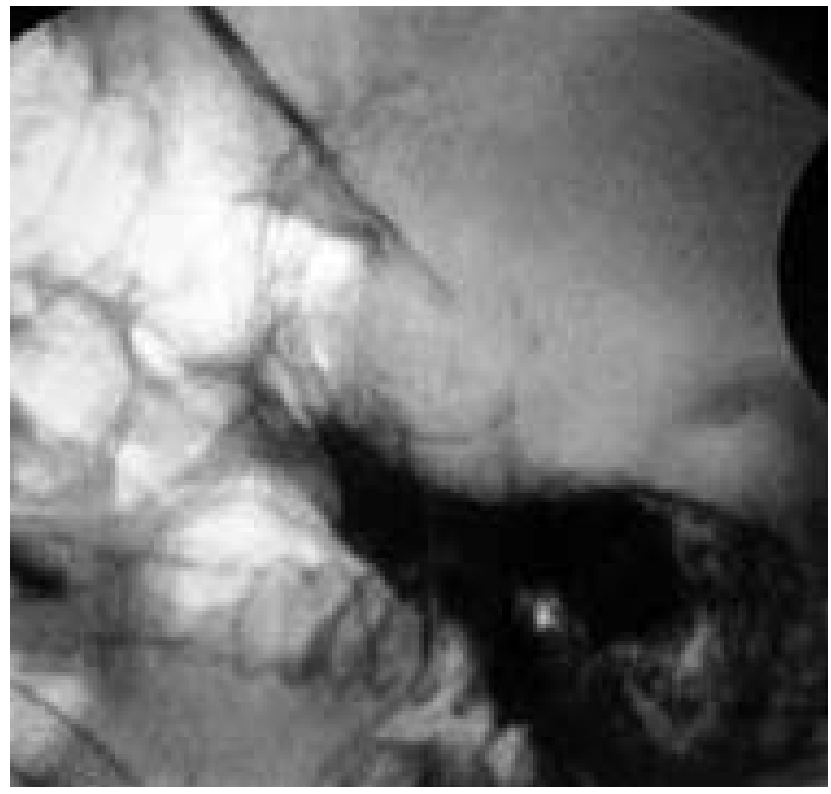

Figura. 1. Caso 1. Rx simple de cráneo: silla turca agrandada y erosionada.

de visión bilateral, agitación y mayor confusión mental. La determinación de Na plasmático fue de $129 \mathrm{mmol} / \mathrm{L}$. Inicia tratamiento con esteroides y se le practica entonces una TAC craneal, siendo remitido a nuestro Servicio con el diagnóstico de hemorragia subaracnoidea.

A su ingreso en nuestro hospital, el paciente tenía una hemianopsia temporal en OD y amaurosis en OI, así como una parálisis del III par derecho y moderada rigidez de nuca, sin ninguna otra focalidad neurológica. La tensión arterial era 120/80 mm Hg. Las pruebas de coagulación fueron normales y la natremia se había corregido a 141 $\mathrm{mmol} / \mathrm{L}$. La diuresis fue normal a lo largo de toda su estancia hospitalaria.

El estudio hormonal, mostró un panhipopituitarismo.

En la Rx de cráneo, la silla turca estaba agrandada y erosionada (Fig. 1). TAC: Masa sellar con invasión supraselar, con una sufusión hemorrágica intratumoral (Fig. 2). En la RNM, la masa tumoral es hipointensa con una porción lateral hiperintensa y nódulo central que capta contraste, comprimiendo las vías ópticas y porción anterior del III ventrículo (Fig. 3).

Se intervino en el sexto día del inicio del cuadro clínico, por vía transesfenoidal, extirpándose un tumor carnoso, rojo-violáceo, con sangrado en sábana, sin que existiera ningún hematoma intratumoral.

El examen anatomopatológico del tumor, mostró una necrosis hemorrágica de adenoma hipofisario.

En el inmediato postoperatorio cursó con una diabetes insípida transitoria e inició una mejoría de sus alteraciones visuales. Seis meses después, el paciente presentaba como secuela, una hemianopsia temporal en OD y una

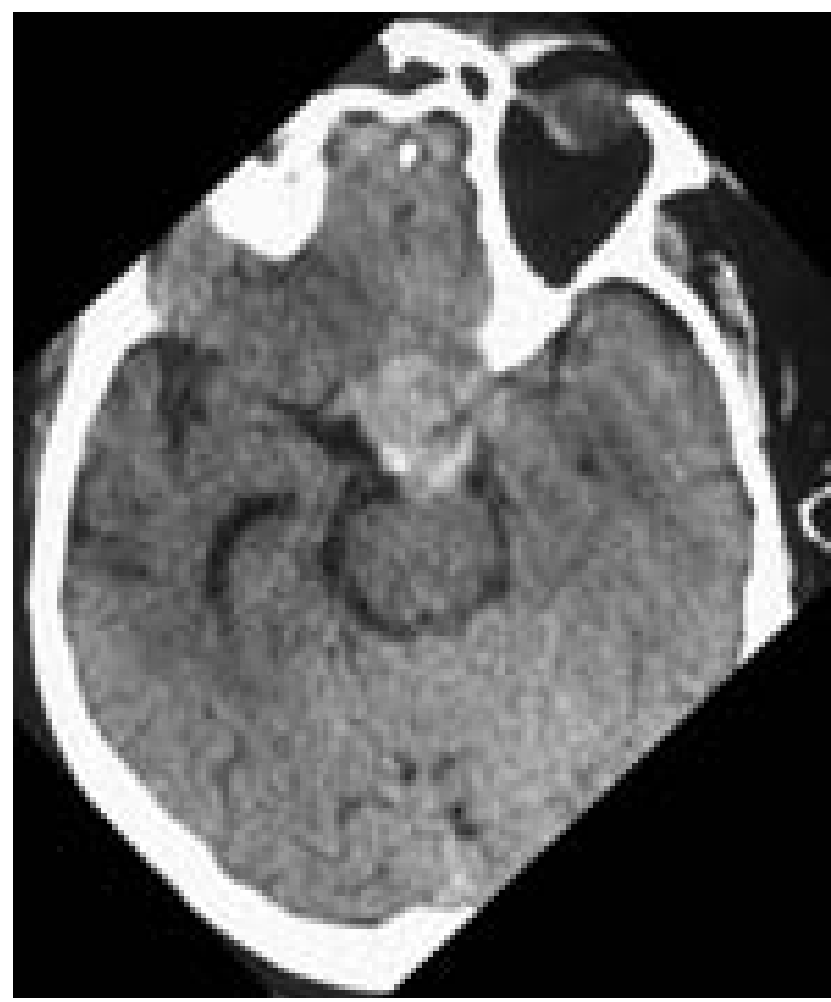

Figura 2. Caso 1: TAC sin contraste: sufusión hemorrágica en la porción supraselar del adenoma.

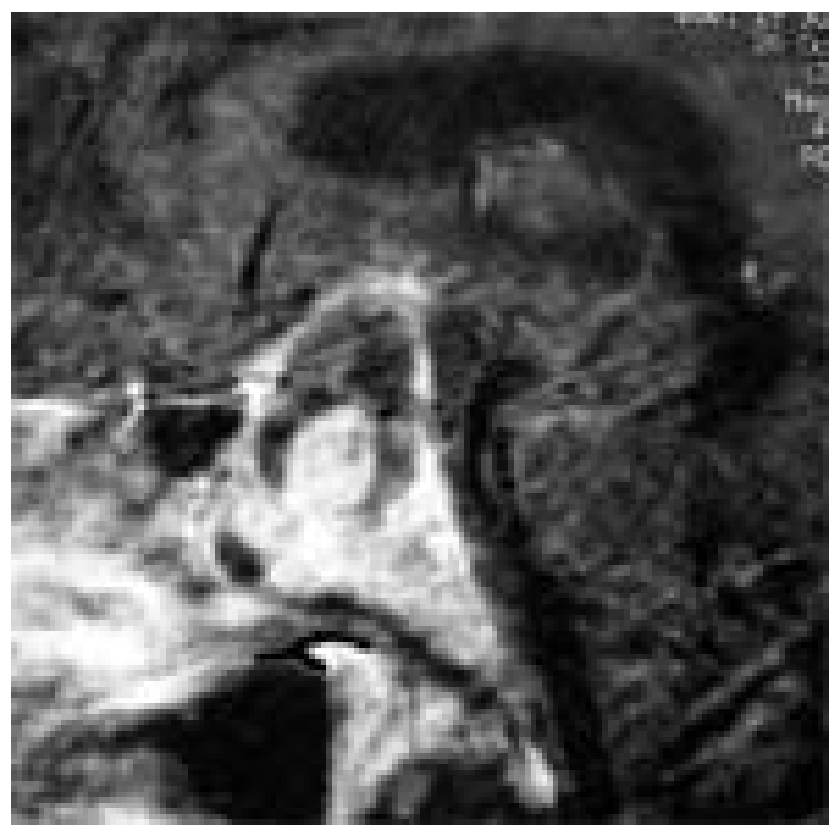

Figura. 3. Caso 1. RNM + contraste: Realce capsular y de un nódulo tumoral central viable.

cuadrantanopsia temporal superior en OI. La RNM mostró la resolución completa del adenoma y, desde el punto de vista endocrinológico, sólo presentaba un déficit del eje gonadotrópico y de hormona de crecimiento. 


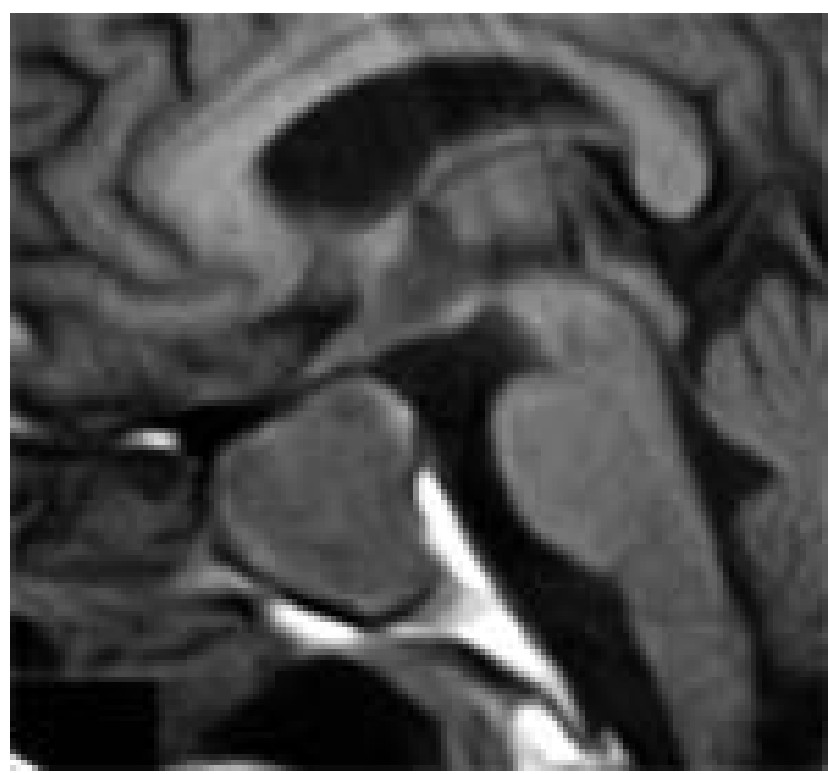

Figura 4. Caso 2: RNM sin contraste. El tumor ha destruido el suelo sellar y ocupa el seno esfenoidal. Existe una indentación diafragmática en la porción superior del tumor.

\section{Caso 2}

Varón de 56 años. Inicia cefalea intensa dos días después de ser operado de la rodilla con anestesia espinal en otro hospital. Cuarenta y ocho horas más tarde del inicio de la cefalea, comienza a ver doble.

Tiene antecedentes de hipertensión arterial en tratamiento, cardiopatía isquémica y de una trombosis de la vena central de la retina en OI hace 8 años, con pérdida grave de la visión por ese ojo donde, además, padece de glaucoma.

En la exploración se apreciaba una agudeza y campo visual normal en OD y una parálisis del III par en ambos lados, con ptosis palpebral casi total.

En la RM se encuentra un tumor hipofisario que agranda y erosiona la silla turca, invade seno esfenoidal y la cisterna supraselar, sin llegar a comprimir las vías ópticas. Es isointenso en T1 (Fig.4), con amplias zonas hiperintensas en T2 (Fig.5).

Los análisis de hormonas en sangre pusieron de manifiesto una elevada cifra de cortisol, debido a que se extrajo la muestra después de haber comenzado el tratamiento con hidrocortisona. El resto de las hormonas hipofisarias era normal.

Quince días después del inicio del cuadro, comienza a mejorar el déficit del III par derecho y se decide mantener una actitud expectante hasta ver la evolución final del tumor, tras el episodio apopléjico. La recuperación oculomotora fue total en el plazo de 3 semanas.

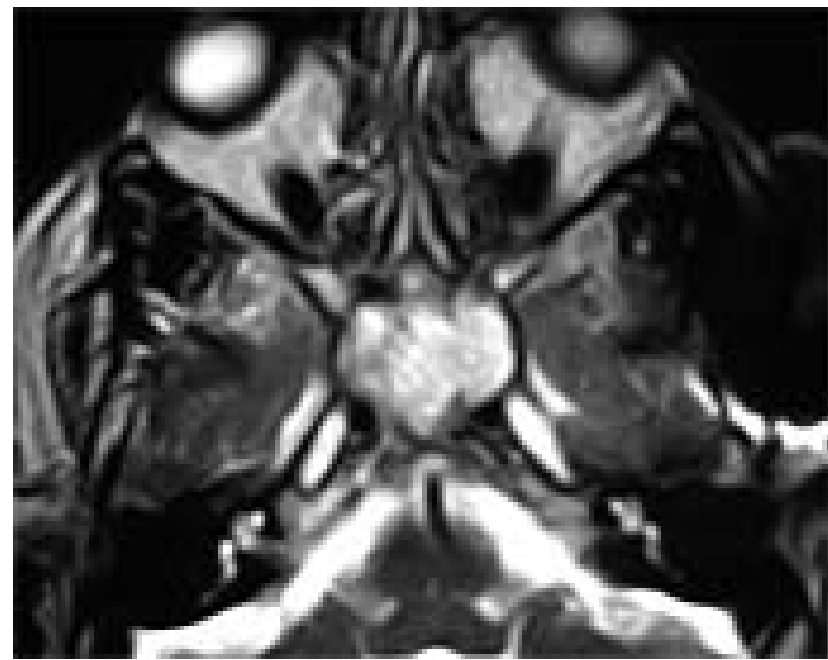

Figura 5. Caso 2: RNM T2 axial. Hiperintensidad tumoral y compresión de los senos cavernosos.

\section{Comentarios}

\section{Consideraciones diagnósticas}

Ambos pacientes sufrieron una apoplejía de un adenoma hipofisario oculto, tras un procedimiento de raquianestesia. El problema es que, si no aparecen síntomas visuales, el cuadro clínico puede ser mal interpretado como una complicación benigna de la propia anestesia espinal y retrasarse el diagnóstico, lo que puede tener consecuencias graves para el enfermo.

En el caso numero $1 \mathrm{y}$ de manera retrospectiva, se constató que sufría una disminución de la potencia sexual desde un año antes, a la que el propio enfermo no le había dado relevancia, creyendo que era un fenómeno propio de la edad. Sin embargo, unido a la presencia de una moderada cefalea de varias semanas de evolución, debería de haber puesto sobre la pista de su patología hipofisaria.

Es conocido que el cuadro clínico de una apoplejía hipofisaria puede ser la primera manifestación de un tumor de

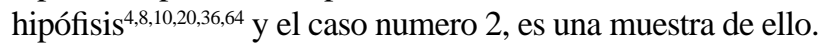

Las dos complicaciones más frecuentes, tras la anestesia espinal, son la cefalea post-punción dural (14\% de los casos y en $7 \%$ en los mayores de 70 años) y la hipotensión arterial $(10-76 \%)^{9,11,21,30}$. La incidencia de bradicardia, se ha publicado en $4,9 \%^{30}$. De manera ocasional puede ocurrir náusea o diplopía por afectación del VI par, así como acúfenos, vértigo y ataxia ${ }^{17}$.

\section{Cefalea tras la anestesia espinal}

La cefalea post-punción lumbar puede comenzar en unas horas, aunque de manera habitual se inicia a las 2448 horas después del procedimiento. Es un dolor frontal u occipital que, a menudo, irradia hacia la nuca y detrás 
de los ojos. Mejora cuando el paciente se acuesta y, de manera típica, empeora al levantarse ${ }^{48}$. El dolor suele durar un promedio de 4 días, aunque puede permanecer varias semanas. Es un dolor causado por la pérdida mantenida de lcr, a través del orificio dejado en la duramadre por la aguja de punción y, cuando excede el ritmo de su producción fisiológica, produce una hipotensión intracraneal y un descenso del encéfalo en relación con la duramadre, como se ha demostrado con $\mathrm{RNM}^{43}$, originando una distensión de estructuras sensibles al dolor como vasos sanguíneos, nervios y paquimeninge. Por lo tanto, una cefalea que aparezca en el periodo de la anestesia espinal, o inmediatamente después, y que no tenga carácter ortostático, debe de hacer sospechar una complicación no relacionada con la habitual hipotensión intracraneal de la punción lumbar.

\section{Parálisis oculo-motoras}

El desarrollo de una parálisis de un par craneal tras una punción lumbar es un hecho, por su frecuencia, raro y es el VI par el afectado en la generalidad de los casos, a veces de forma bilateral ${ }^{26,60}$. Su lesión se atribuye, tras la hipotensión intracraneal, a la tracción que ejerce sobre el nervio el descenso del tronco cerebral, provocando su neuropraxia o axonotmesis. Este mecanismo está favorecido por el anclaje que tiene el nervio a la punta del peñasco y por tener una orientación ascendente en su recorrido cisternal.

El calibre de la aguja empleada en la punción lumbar tiene relación con la cantidad de lcr que se escapa por el orificio que deja la aguja en la duramadre y, por tanto, con la intensidad del cuadro de hipotensión intracraneal. Dripps RD y col ${ }^{17}$, han publicado 8 casos de parálisis del VI par en 839 pacientes que recibieron anestesia espinal con aguja del $16 \mathrm{G}$, en contraposición con ninguno en 7654 pacientes que recibieron anestesia espinal con agujas del 20-22 G. Thömke y $\mathrm{col}^{60}$, con su experiencia en 11.600 pacientes, deducen un riesgo de menos de 1 entre 5800 punciones lumbares, si se emplea una aguja del $22 \mathrm{G}$.

La afectación de otros pares craneales tras una punción lumbar, es francamente excepcional. Nishio y $\mathrm{col}^{40}$, en una revisión de 95 casos de parálisis motoras oculares tras anestesia espinal, publicados desde 1966 al 2002, encontraron sólo cuatro pacientes con afectación del III par, dos pacientes con afectación del IV par y uno con lesión del V par, siendo el resto lesiones del VI par.

La parálisis ocular se manifiesta de 1 día a 3 semanas después de haber realizado la punción lumbar, con una media de 6 días. Generalmente es precedida por la cefalea aunque, a veces, puede aparecer antes de que ésta se inicie o después de que el dolor de cabeza haya remitido. La recuperación de los pares craneales es espontánea en el plazo de 2 semanas a 8 meses en el $89 \%$ de los pacientes, la mayoría dentro de los seis meses ${ }^{40}$.

En contraposición a la incidencia de complicaciones motoras oculares en la anestesia espinal, la parálisis del III par en la apoplejía de un adenoma hipofisario con expresión clínica, es muy frecuente. En nuestra experiencia con 29 casos de apoplejía hipofisaria, la lesión del nervio oculomotor afectó a 11 de ellos (37,9\%), estando asociada a una parálisis del VI par en 2 enfermos. La parálisis aislada del VI par sólo la sufrieron 3 pacientes. Por lo tanto, la aparición de un tercer par en el contexto de una anestesia espinal, debe de hacer sospechar siempre la posibilidad de una apoplejía hipofisaria, como pone en evidencia los dos casos que exponemos.

\section{Meningitis aséptica y alteración del sensorio}

Otra complicación neurológica benigna de la anestesia espinal, es la meningitis aséptica ${ }^{21}$. Se presenta generalmente dentro de las 24 horas de la anestesia espinal y se caracteriza por fiebre, rigidez de nuca y fotofobia, síntomas que, a veces, pueden ser compartidos por la apoplejía hipofisaria. El examen del lcr en estas meningitis asépticas, muestra una leucocitosis polimorfonuclear con cultivo bacteriológico negativo. Síntomas como confusión mental y niveles más alterados de consciencia, como sucedió en nuestro primer paciente, son inusuales tras la anestesia espinal y deben de hacer pensar, entre otras posibilidades, en un infarto del tronco cerebral o en una hiponatremia secundaria a insuficiencia suprarrenal aguda ${ }^{16,20,23,35,68}$ que, con frecuencia, acompaña a la apoplejía hipofisaria. En estos casos, es imperativo hacer el diagnostico precoz del verdadero motivo por el que el paciente se está deteriorando, para iniciar el tratamiento sustitutivo esteroideo necesario para estabilizar al enfermo.

\section{Hematoma subdural}

El hematoma subdural, como consecuencia de la hipotensión intracraneal y descenso del cerebro, resultando en desgarro de las venas puente de la convexidad cerebral, es un hecho raro pero bien conocido como complicación de la punción lumbar voluntaria o inadvertida ${ }^{14,37,63,67,72}$. También ha sido publicado en hipotensión intracraneal espontánea $^{15,54}$. De manera excepcional puede cursar de forma aguda $^{3,67}$, pero lo más frecuente es la instauración insidiosa con expresión clínica a partir de los 15-20 días del procedimiento ${ }^{14,29,45,56,63}$, pudiendo haber desaparecido ya la cefalea post-punción o cambiar su carácter ortostático. También la punción lumbar, puede agravar un hematoma subdural crónico preexistente ${ }^{54,71}$. En cualquier caso, ya sea la presentación precoz o tardía, la ausencia del carácter ortostático de la cefalea, la aparición, bien aislada o en combinación, de una focalidad en las vías largas o alteraciones de consciencia, en mayor o menor grado, en estos pacientes, deben de hacer pensar en la posibilidad de un hematoma subdural y, la afectación del III par craneal, implica que el hematoma está evolucionando hacia una hernia cerebral 
transtentorial, lo que constituye también una emergencia neuroquirúrgica.

\section{Rotura de un aneurisma}

El inicio o exacerbación brusca de una cefalea, rigidez de nuca, diferentes grados de alteración de la consciencia y una parálisis del III par, como sucedió en el primer caso que presentamos, es una clínica que pueden compartir tanto la apoplejía hipofisaria, como la rotura de un aneurisma. Este último, en el contexto de una anestesia espinal, puede ser considerado como puramente casual, si bien se ha especulado sobre si la hipotensión intracraneal tras la punción lumbar, puede favorecer la rotura del aneurisma, al aumentar el gradiente transmural a nivel de su pared.

\section{Esclerosis múltiple}

Otra complicación inusual de la anestesia espinal es la aparición de una parálisis oculomotora, como primera manifestación de una esclerosis múltiple hasta entonces asintomática $^{34}$, lo que pudiera ser debido a la acción del propio anestésico, al alcanzar estructuras más altas del SNC, poniendo en evidencia una placa de desmielinización enmascarada ya preexistente ${ }^{44}$.

\section{Consideraciones sobre la patogenia de la apoplejía en la anestesia espinal}

El mecanismo por el que una anestesia espinal puede desencadenar la apoplejía hipofisaria de un adenoma preexistente, es especulativa. Pero existen datos que nos permiten hacer una aproximación teórica.

\section{Vascularización de la hipófisis normal}

La adenohipófisis, en contraposición a la neurohipófisis, no recibe irrigación arterial directa ${ }^{42,57,61,66}$ (Fig. 6), con excepción de pequeñas ramitas arteriales a partir de las arterias lorales de Stanfield ${ }^{57}$ (arteria de la trabecula de Xuereb ${ }^{66}$ ), o procedentes de la cápsula dural, cuya sangre sólo alcanza las capas más periféricas de la glándula. El principal aporte sanguíneo, proviene de los vasos porta, de naturaleza venosa, que transporta la sangre desde la eminencia media y tallo hipofisario, hasta los sinusoides adenohipofisarios que, ampliamente comunicados entre sí, distribuyen la sangre por toda la glándula y la mantienen bajo control hormonal hipotalámico.

El sistema porta es un sistema de baja presión. Findell y $\mathrm{col}^{18}$, en estudios experimentales en ratas, obtuvieron una presión media de 2.7 mm Hg en registros obtenidos en la luz de los vasos porta largos. Kemeny y col$^{24}$, demostraron la existencia de una autorregulación en el sistema, que lo hace estable entre variaciones de la presión arterial de 40 y $140 \mathrm{~mm}$ de $\mathrm{Hg}$, en las porciones centrales de adenohipófisis, y entre 40-170 mm de Hg en las porciones
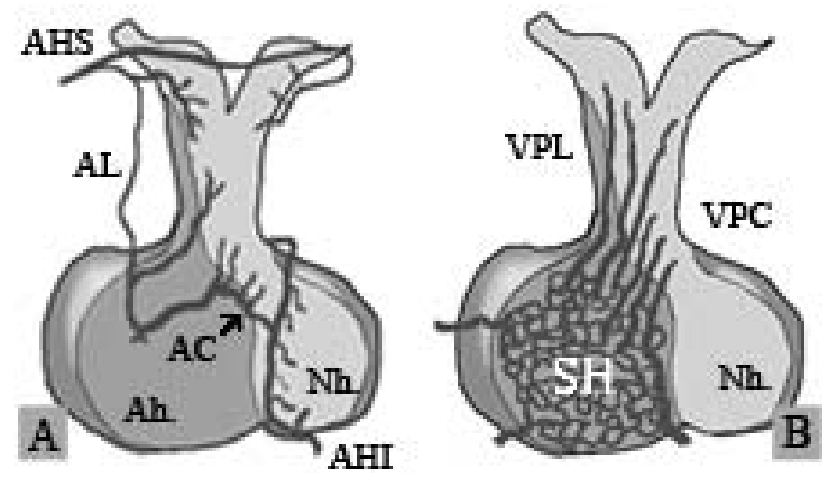

Figura 6. Esquema de la irrigación sanguínea de la hipófisis (Modificado de Xuereb ${ }^{66}$ ). A: arterias de la hipófisis. La arteria de la trabecula, solo da unos pequeñas ramitas a la adenohipófisis, pese a que pasa a través de ella en parte de su recorrido. Las arterias capsulares no están representadas. B: irrigación de la adenohipófisis por el sistema porta. AC: rama arterial comunicante. Ah: adenohipófisis AHS: arterias hipofisarias superiores. AHÍ: arterias hipofisarias inferiores. AL: arteria loral o de la trabecula. Nh: neurohipófisis. SH: sinusoides de la adenohipófisis. VPC: vasos porta cortos. VPL: vasos porta largos.

laterales, de manera que se mantiene un adecuada perfusión de la adenohipófisis, con presiones arteriales medias tan bajas como $40 \mathrm{~mm} \mathrm{Hg}$. de manera que, aún siendo un sistema vulnerable a los descensos graves de la presión arterial, debe de haber otros factores coincidentes que potencien los efectos adversos de una disminución menos drástica de esta.

\section{Vascularización de los adenomas}

En la mayoría de los tumores hipofisarios, se ha demostrado que existe una neo-vascularización arterial ${ }^{6,19,47,50,62,69}$, independiente del sistema porta. Un estudio angiográfico, demostró vasos tumorales que se originaban directamente del sistema arterial, a través de las arterias hipofisarias inferiores $^{46}$. Estudiando la secuencia de captación de contraste con RM, los adenomas se realzan inmediatamente después de la neurohipófisis y antes que la adenohipófisis, lo que es congruente con una irrigación independiente del sistema porta $^{69}$. Pese a esa angiogénesis, los tumores hipofisarios tienen una vascularización más pobre que las hipófisis normales ${ }^{22,62}$. Ya en 1950, Brougham y col. ${ }^{7}$-que fueron los que por primera vez acuñaron el término de apoplejía hipofisaria-, sugirieron que algunos de estos tumores crecen por encima de sus posibilidades de vascularización, lo que les lleva al infarto y a la hemorragia.

Los vasos tumorales de los adenomas de hipófisis suelen ser más pequeños que los de la glándula normal. Con microscopía electrónica tienen rasgos de maduración incompleta, con escasas fenestraciones. Las membranas 
basales están, a menudo, rotas y fragmentadas ${ }^{8}$. Estas características hacen suponer que la vascularización de los adenomas es frágil, lo que está en congruencia con el hecho de que sangran con una frecuencia 5,4 veces mayor que otros tumores cerebrales ${ }^{65}$. Como los aneurismas intracraneales se asocian a los adenomas hipofisarios con más frecuencia que la población general, se ha especulado sobre la existencia de algún tipo de vasculopatía como mecanismo subyacente común a la formación de aneurismas y de la apoplejía del adenoma hipofisario ${ }^{8}$.

\section{Presión intraselar en los adenomas de hipófisis}

Los estudios de presión intraselar, en los adenomas de hipófisis, han mostrado cifras medias, como grupo, en el rango de 23 a $30 \mathrm{~mm} \mathrm{Hg}^{2,28,31,32}$, habiéndose medido presiones tan altas como $51 \mathrm{~mm} \mathrm{Hg}^{32}$ y $62 \mathrm{~mm} \mathrm{Hg}^{28}$ lo que, sin duda, compromete la presión de perfusión de un tejido que estuviera exclusivamente irrigado por el sistema porta. Aunque la angiogénesis tumoral, aportando de manera directa sangre arterial, sea una ayuda para la supervivencia celular, es un inconveniente para el control hormonal hipotalámico de la hipófisis, habiéndose propuesto este mecanismo para justificar las disfunciones hormonales, en especial la hiperprolactinemia, clásicamente atribuida al efecto compresivo del tumor sobre el tallo hipofisario ${ }^{32}$. En cualquiera de los casos, la perfusión del tejido tumoral debe de tener un margen pequeño, ya que periódicamente se producen fenómenos de apoplejía tumoral, en donde se ha obtenido una mediana de la presión intraselar de $47 \mathrm{~mm} \mathrm{Hg}^{70}$.

\section{Tamaño del adenoma e hipotensión intracraneal}

Rovit y $\mathrm{col}^{49}$ propusieron como mecanismo de la apoplejía en los macroadenomas la compresión que puede hacer el tejido tumoral, en su crecimiento a través del orificio diafragmático, sobre las arterias de la trabécula y los vasos porta del tallo hipofisario, yugulando el principal aporte sanguíneo de la hipófisis. Esta teoría ha sido criticada en base, por un lado, a la demostración angiográfica de que los adenomas reciben su vascularización a través de las arterias hipofisarias inferiores, como se ha comentado antes y, por otro lado, a que también se producen apoplejías en tumores pequeños que no han crecido a través del orificio diafragmático. Sin embargo, puede ser un mecanismo válido en determinados pacientes, ya que nosotros no creemos que exista un mecanismo universal que justifique todas las apoplejías hipofisarias, sino más bien una concurrencia de factores que, en un paciente en particular, desencadena el fenómeno apopléctico y estos factores pueden variar de un enfermo a otro. Los estudios angiográficos no son determinantes y se ha puesto en duda de si el realce que demuestran es capsular o del parénquima tumoral ${ }^{8}$. En cualquier caso, la estrangulación de la circulación sanguínea por las aferencias del sistema porta o por el mismo sistema porta, lleva inevitablemente al infarto del tejido adenohipofisario remanente normal ${ }^{13}$ y esto puede desencadenar un aumento brusco, adicional, de la presión intraselar y colapsar la precaria irrigación sanguínea del tumor, lo que llevaría a cerrar el círculo de acontecimientos.

La hipotensión intracraneal tras punción lumbar -una de las complicaciones más frecuentes de la anestesia espinal- puede, al menos en teoría, alterar el gradiente de presión entre la fosa sellar -con una presión alta- y la cavidad craneana, terminando por impactar el tumor contra el orificio diafragmático de la silla turca, desencadenando así los fenómenos que llevan al infarto tisular. En nuestro primer caso, así como en el de Lennon y col., quizás el inicio del cuadro fue demasiado precoz para tener en cuenta este mecanismo, pero no así en nuestro segundo paciente, cuya cefalea se inició a las 48 horas de la intervención quirúrgica, tiempo suficiente para haberse desarrollado una hipotensión intracraneal. Sin embargo, y hasta ahora, no hemos encontrado ningún artículo que refiera este hecho en la bibliografía, al menos sin que concurran otros factores que puedan estar potencialmente implicados, como es la antigua neumoencefalografía ${ }^{8}$.

Por otro lado se ha demostrado mediante estudios de RM que, en los cuadros de hipotensión intracraneal, la hipófisis aumenta su tamaño de forma anormal ${ }^{1,39,55}$, volviendo a sus dimensiones normales después de normalizarse la presión del lcr, lo que se ha atribuido a una hipervolemia pituitaria secundaria al aumento de volumen sanguíneo intracraneal -en acorde con la doctrina de Monro-Kellie- que se refleja de manera primaria en la dilatación de los senos venosos y venas meníngeas ${ }^{39,52}$. Esto podría causar un drenaje venoso de la hipófisis más lento y/o un aumento adicional de la presión intraselar, desencadenando la apoplejía del adenoma. De nuevo aquí cabría esperar un margen de tiempo racional, tras la práctica de la anestesia espinal, para que se instaure el cuadro de hipotensión intracraneal, lo que sólo se ha producido, de los tres casos, en nuestro segundo paciente.

\section{Estimulación de la adenohipófisis}

Se ha sugerido que el estrés quirúrgico somete a la hipófisis a un estímulo excesivo para producir grandes cantidades de esteroides y esta demanda metabólica, al no estar acompañada de una irrigación sanguínea apropiada en los adenomas grandes, llevaría al infarto y a la hemorragia tumoral $^{5}$. Este hecho se basa en los casos publicados de apoplejías en pacientes sometidos a otras situaciones de estimulación hipofisaria, como la que se produce en el embarazo, por los estrógenos, o durante las pruebas de estímulo hipofisario, para averiguar la reserva funcional de la glándula ${ }^{5,51}$. También en la apoplejía que aparece durante un infarto de miocardio o durante una intervención quirúrgica, pero en estos últimos existen otros factores que pueden contribuir y potenciar los efectos adversos de la 




Figura 7. Extenso infarto adenohipofisario no tumoral. Observación personal (JGT). En la zona que rodea al núcleo central necrótico, existe una zona (ZPI) en la que sólo el endotelio de los vasos ha sobrevivido y pueden contener hematíes por dos o tres días ${ }^{53}$. Por fuera de esta zona periférica, existe una capa viable de células adenohipofisarias (ZS), en inmediata vecindad con la neurohipófisis.

estimulación hipofisaria, como veremos a continuación.

Sin embargo, si el adenoma tiene una irrigación arterial directa, queda apartado durante el estrés quirúrgico, al menos en parte, del influjo estimulador hipotalámico, por lo que es más probable que el infarto se produzca en el tejido hipofisario normal que aún permanezca bajo la influencia del hipotálamo, a través de los vasos porta.

\section{Hipotensión arterial}

La hipotensión arterial es uno de los factores etiológicos bien conocidos y documentados del infarto isquémico de la adenohipófisis. En los casos más graves de shock vascular por parto ${ }^{53}$, todo el parénquima adenohipofisario, cuya irrigación depende exclusivamente de los vasos del sistema porta, se necrosa (Fig. 7); es decir, el 97-99\% de la adenohipófisis, dependiendo de la intensidad y duración de la hipotensión arterial. Quedan respetados el tallo hipofisario, neurohipófisis y pequeñas zonas adenohipofisarias que pueden recibir alguna irrigación arterial directa entre las que, de manera típica, se incluyen una o dos capas celulares adyacentes a la cápsula glandular (Fig. 8). Cuando la hipotensión se recupera y la circulación se restablece puede haber una hemorragia secundaria, a partir de los vasos que han resultado dañados en el proceso de isquemia.

Como se ha comentado antes, una de las complicaciones más frecuentes de la anestesia espinal, es la hipotensión arterial. Chinachoti y $\mathrm{col}^{9}$, tomando como referencia de hipotensión un descenso igual o mayor del $20 \%$ de la presión sistólica, publican una incidencia del 57,9\% entre 2000 pacientes sometidos a anestesia espinal. Chumpathong y

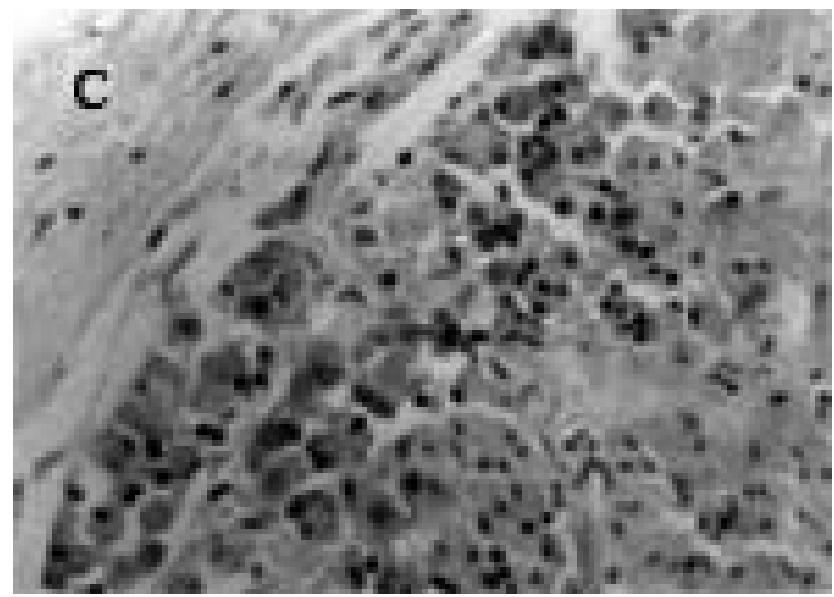

Figura 8. Imagen típica de células adenohipofisarias viables subcapsulares en otro caso de infarto adenohipofisario no tumoral. Observación personal (JGT).

$\mathrm{col}^{11}$, tuvieron una incidencia del 76\%, tomando como referencia una tensión arterial sistólica de 100 mm Hg o menos. Es decir, entre los pacientes sometidos a anestesia espinal, la hipotensión arterial es extraordinariamente frecuente y el primer caso que presentamos es una muestra de ello. Desafortunadamente, no disponemos de información referente a la evolución de la presión sanguínea en nuestro segundo caso, pero en la apoplejía publicada por Lennon y $\mathrm{col}^{33}$, el paciente sufrió un descenso de la TA sistólica desde la inicial de $120 \mathrm{~mm} \mathrm{Hg}$, hasta $90 \mathrm{~mm} \mathrm{Hg}$ y se mantuvo en este nivel durante todo el tiempo de la intervención quirúrgica.

Es obvio que el descenso de la tensión arterial, en la anestesia espinal, es un descenso moderado. Para que pueda provocar un infarto glandular es necesaria la concurrencia de los otros factores condicionantes: una irrigación del tejido intraselar previamente comprometida y, probablemente, una mayor demanda metabólica de la glándula por el estrés quirúrgico.

La mayoría de apoplejías tumorales hipofisarias, publicadas en el contexto de una intervención quirúrgica, se han producido tras sufrir el paciente un tiempo de hipotensión arterial moderada. Cohen y $\mathrm{col}^{12}$, publicaron un caso tras cirugía para liposucción abdominal, en la que la TA del paciente cayó desde 120 a $90 \mathrm{~mm} \mathrm{Hg}$, permaneciendo en este nivel durante 30 minutos. La cirugía cardiaca, en especial con bypass cardiopulmonar, es la que acumula la mayor incidencia. En ellos se ha considerado que la ausencia de flujo pulsátil que acompaña al bypass cardiopulmonar no-pulsátil, agrava los efectos deletéreos de la hipotensión arterial $^{38}$. Otros factores que están presentes en esta cirugía cardiaca y que pueden influir en el desarrollo de la apoplejía hipofisaria -no en la anestesia espinal- son, la terapéutica anticoagulante, la dilución sanguínea y descenso del hematocrito, -causado por la solución de cristaloide con la 
que habitualmente se ceba la bomba- y la propensión al desarrollo de microembolias por el bypass cardiopulmonar y la manipulación cardiaca o aórtica ${ }^{38}$. Estos factores concurrentes en la cirugía cardiaca mayor no precisan de la existencia de un tumor que aumente la presión intraselar y tenga comprometida su vascularización, ya que Kovacs y $\mathrm{Yao}^{27}$, en un estudio de 33 pacientes fallecidos en los 10 días siguientes a una cirugía cardiaca mayor, encontraron necrosis agudas de la glándula en el 15,1\% de los casos. En contraposición, en un estudio de 55 autopsias correlativas de pacientes sin patología tumoral hipofisaria, uno de los autores de este artículo (GT), encontró solo un 5,4\% de infartos adenohipofisarios.

\section{Hipertensión arterial}

Para los pacientes que padecen hipertensión arterial crónica, la hipotensión arterial es un factor de riesgo de infarto tisular ${ }^{58}$, ya que en ellos el límite del descenso de la TA para que no falle la autorregulación cerebral está desplazado hacia un nivel más alto, con respecto a las personas con TA normal ${ }^{59}$. Además, en estos enfermos existe una disminución, focal o difusa, de la perfusión cerebral ${ }^{41}$, en especial en los que no reciben tratamiento. Desconocemos la influencia de la hipertensión arterial esencial, como factor agravante de la hipotensión arterial de la anestesia espinal, en los macroadenomas de hipófisis. Nuestros dos pacientes sufrían de hipertensión arterial esencial, pero no así el caso publicado por Lennon y $\operatorname{col}^{33}$. También la bradicardia puede agravar los efectos indeseados de la hipotensión arterial y tanto nuestro primer caso, como el de Lennon y col, la padecieron.

Considerando todas las circunstancias comentadas anteriormente, es probable que la hipotensión arterial que, con frecuencia, acompaña a la anestesia espinal y que hemos podido objetivar en el caso $\mathrm{n}^{\mathrm{o}} 1 \mathrm{y}$ en el publicado por Lennon y col ${ }^{33}$, sea el factor desencadenante del infarto del macroadenoma de hipófisis en estos enfermos, al descompensar la precaria irrigación sanguínea arterial del tumor, previamente comprometida por el aumento de la presión intraselar.

El factor desencadenante de la apoplejía hipofisaria en el segundo paciente que presentamos, cuyos síntomas se iniciaron dos días después de ser operado, podría haber sido el desarrollo de una hipotensión intracraneal secundaria a la punción lumbar pero carecemos de datos objetivos que sustenten esta hipótesis.

\section{Conclusiones}

La aparición de una parálisis del III par craneal, en el contexto de una cefalea post-anestesia espinal de inicio precoz, en especial si ésta pierde en su evolución, o no tiene desde el principio, el típico carácter ortostático de la cefalea post-punción lumbar, debe de hacer pensar en la apoplejía de un macroadenoma de hipófisis oculto y, cuando menos, ser el inicio de exploraciones complementarias urgentes que posibiliten su diagnóstico precoz y el tratamiento específico.

Es probable que la hipotensión arterial, durante el procedimiento de anestesia espinal, sea el mecanismo desencadenante más significativo de la apoplejía de un macroadenoma preexistente, pero debe de ser confirmado con una casuística más numerosa.

\section{Bibliografía}

1. Alvarez-Linera, J., Escribano, J., Benito-León, J., PortaEtessam, J., Rovira, A.: Pituitary enlargement in patients with intracranial hypotension syndrome. Neurology. 2000 Dec 26; 55: 1895-1897.

2. Arafah, B.M., Prunty, D., Ybarra, J., Hlavin, M.L., Selman, W.R.: The dominant role of increased intrasellar pressure in the pathogenesis of hypopituitarism, hyperprolactinemia, and headaches in patients with pituitary adenomas. J Clin Endocrinol Metab. 2000 May; 85: 1789-1793.

3. Béal, J.L., Royer, J.M., Freysz, M., Poli, L., Wilkening, M.: Acute intracranial subdural hematoma of arterial origin after spinal anesthesia. Ann Fr Anesth Reanim. 1989; 8:143-145.

4. Bills, D.C., Meyer, F.B., Laws, E.R. Jr., Davis, D.H., Ebersold, M.J., Scheithauer, B.W., Ilstrup, D.M., Abboud, C.F.: A retrospective analysis of pituitary apoplexy. Neurosurgery. 1993 Oct; 33: 602-608; discussion 608-609.

5. Biousse, V., Newman, N.J., Oyesiku, N.M.: Precipitating factors in pituitary apoplexy. J Neurol Neurosurg Psychiatry 2001; 71: 542-545.

6. Bonneville, J.F., Cattin, F., Gorczyca, W., Hardy, J.: Pituitary microadenomas: early enhancement with dynamic CT--implications of arterial blood supply and potential importance. Radiology. 1993 Jun; 187: 857-861

7. Brougham, M., Heusner, A.P., Adams, R.D.: Acute degenerative changes in adenomas of the pituitary body--with special reference to pituitary apoplexy. J Neurosurg. 1950 Sep; 7: 421-439.

8. Cardoso, E.R., Peterson, E.W.: Pituitary apoplexy: a review. Neurosurgery. 1984 Mar; 14: 363-673.

9. Chinachoti, T., Tritrakarn, T.: Prospective study of hypotension and bradycardia during spinal anesthesia with bupivacaine: incidence and risk factors, part two. J Med Assoc Thai. 2007 Mar; 90: 492-501.

10. Chuang, C.C., Chang, C.N., Wei, K.C., Liao, C.C., Hsu, P.W., Huang, Y.C., Chen, Y.L., Lai, L.J., Pai, P.C,: Surgical treatment for severe visual compromised patients after pituitary apoplexy. J Neurooncol. 2006 Oct; 80: 39-47.

11. Chumpathong, S., Chinachoti, T., Visalyaputra, S., Himmunngan, T.: Incidence and risk factors of hypotension during spinal anesthesia for cesarean section at Siriraj Hospi- 
tal. J Med Assoc Thai. 2006 Aug; 89: 1127-1132.

12. Cohen, A., Kishore, K., Wolansky, L., Frohman, L.: Pituitary apoplexy occurring during large volume liposuction surgery. J Neuro-Ophthalmol 2004. 24: 31-33.

13. Daniel, P.M., Prichard, M.M.: Anterior pituitary necrosis; infarction of the pars distalis produced experimentally in the rat. Q J Exp Physiol Cogn Med Sci. 1956 Jul; 41: 215-229.

14. Davies, J.M., Murphy, A., Smith, M., O'Sullivan, G.: Subdural haematoma after dural puncture headache treated by epidural blood patch. Br J Anaesth. 2001 May; 86: 720-723.

15. de Noronha, R.J., Sharrack, B., Hadjivassiliou, M., Romanowski, C.A.J.: Subdural haematoma: a potentially serious consequence of spontaneous intracranial hypotension. Journal of Neurology Neurosurgery and Psychiatry 2003; 74:752-755.

16. Diederich, S., Franzen, N.F., Bähr, V., Oelkers, W.: Severe hyponatremia due to hypopituitarism with adrenal insufficiency: report on 28 cases. Eur J Endocrinol. 2003 Sep; 149: 177-178.

17. Dripps, R.D., Vandam, L.D.: Long-term follow-up of patients who received 10,098 spinal anesthetics. JAMA 1954; 156: 1486-1491.

18. Findell, P.R., Mulchahey, J.J., Shepard, J.M., Gropper, M.A., Battacharya, J., Weiner, R.I.: Direct measurement of blood pressure within the long hypophysial portal blood vessels. Neuroendocrinology. 1987 Apr; 45: 263-266.

19. Gorczyca, W., Hardy, J.: Microadenomas of the human pituitary and their vascularization. Neurosurgery. 1988 Jan; 22: 1-6.

20. Greenberb, M.S.: Pituitary adenomas En Mark S. Greenberg (ed). Handbook of Neurosurgery. Fifth edition. Stuttgart (Germany). Thieme International. 2001; pp: 419.

21. Hyderally, H.: Complications of spinal anesthesia. Mt Sinai J Med. 2002 Jan-Mar; 69: 55-56.

22. Jugenburg, M., Kovacs, K., Stefaneanu, L., Scheithauer, B.W.: Vasculature in Nontumorous Hypophyses, Pituitary Adenomas, and Carcinomas: A Quantitative Morphologic Study. Endocr Pathol. 1995 Summer; 6: 115-124.

23. Kamoi, K., Tamura, T., Tanaka, K., Ishibashi, M., Yamaji, T.: Hyponatremia and osmoregulation of thirst and vasopressin secretion in patients with adrenal insufficiency. J Clin Endocrinol Metab. 1993 Dec; 77: 1584-1588.

24. Kemeny, A.A., Jakubowski, J.A., Jefferson, A.A., Pasztor, E.: Blood flow and autoregulation in rat pituitary gland. J Neurosurg. 1985 Jul; 63: 116-119.

25. Kohara, K., Jiang, Y., Igase, M., Takata, Y., Fukuoka, T., Okura, T., Kitami, Y., Hiwada, K.: Postprandial hypotension is associated with asymptomatic cerebrovascular damage in essential hypertensive patients. Hypertension. 1999 Jan; 33:565-568.

26. Kose, K.C., Cebesoy, O., Karadeniz, E., Bilgin, S.: Eye problem following foot surgery--abducens palsy as a complication of spinal anesthesia. MedGenMed. 2005; 7: 16.

27. Kovacs, K., Yao, J.: Pituitary necrosis following major heart surgery. Z Kardiol. 1975 Jan; 64: 52-57.
28. Kruse, A., Astrup, J., Cold, G.E., Hansen, H.H.: Pressure and blood flow in pituitary adenomas measured during transsphenoidal surgery. Br J Neurosurg. 1992; 6: 333-341.

29. Kunz, U., Panning, B., Stolke, D.: Chronic subdural hematoma following spinal anesthesia. Reg Anaesth. 1989 Mar; 12: 34-37.

30. Kyokong, O., Charuluxananan, S., Sriprajittichai, P., Poomseetong, T., Naksin, P.: The incidence and risk factors of hypotension and bradycardia associated with spinal anesthesia. J Med Assoc Thai. 2006 Sep; 89 Suppl 3: S58-64.

31. Lees, P.D., Fahlbusch, R., Zrinzo, A., Pickard, J.D.: Intrasellar pituitary tissue pressure, tumour size and endocrine status--an international comparison in 107 patients. Br J Neurosurg. 1994; 8: 313-318.

32. Lees, P.D., Pickard, J.D.: Hyperprolactinemia, intrasellar pituitary tissue pressure, and the pituitary stalk compression syndrome. J Neurosurg. 1987 Aug; 67: 192-196.

33. Lennon, M., Seigne, P., Cunningham, A.J.: Pituitary apoplexy after spinal anaesthesia. Br J Anaesth. 1998 Oct; 81: 616-618.

34. Levesque, P., Marsepoil, T., Venutolo, P.H., Lesouef, J.M.: Multiple sclerosis revealed by spinal anesthesia.

35. Lin, S.H., Hung, Y.H., Lin, Y.F.: Severe hyponatremia as the presenting feature of clinically non-functional pituitary adenoma with hypopituitarism. Clin Nephrol. 2002 Jan; 57:85-88.

36. Lubina, A., Olchovsky, D., Berezin, M., Ram, Z., Hadani, M., Shimon, I.: Management of pituitary apoplexy: clinical experience with 40 patients. Acta Neurochir (Wien). 2005 Feb; 147: 151-157.

37. Mashour, G.A., Schwamm, L.H., Leffert, L.: Intracranial subdural hematomas and cerebral herniation after labor epidural with no evidence of dural puncture. Anesthesiology. 2006 Mar; 104: 610-612.

38. Mattke, A.F., Vender, J.R., Anstadt, M.P.: Pituitary Apoplexy Presenting as Addisonian Crisis. Tex Heart Inst J 2002; 29: 193-199.

39. Mokri, B., Atkinson, J.L.: False pituitary tumor in CSF leaks. Neurology. 2000 Aug 22; 55: 573-575.

40. Nishio, I., Williams, B.A., Williams, J.P.: Diplopia. A Complication of Dural Puncture. Anesthesiology 2004; 100:158-164.

41. Nobili, F., Rodriguez, G., Marenco, S., De Carli, F., Gambaro, M., Castello, C., Pontremoli, R., Rosadini, G.: Regional cerebral blood flow in chronic hypertension. A correlative study. Stroke. 1993 Aug; 24: 1148-1153.

42. Page, R.B., Bergland, R.M.: Pituitary vasculature, in Allen MB Jr, Mahesh VB (eds): The Pituitary: a Current Review. New York: Academic Press, 1977, pp 9-17.

43. Pannullo, S.C., Reich, J.B., Krol, G., Deck, M.D., Posner, J.B.: MRI changes in intracranial hypotension. Neurology. 1993; 43: 919-926.

44. Perlas, A., Chan, V.W.: Neuroaxial anesthesia an multiple sclerosis. Can J Anesth 2005; 52: 454-458.

45. Petrognani, R., Hovette, P., Barberet, G., Badiane, C., 
Camara, P., Burgel, P., Theobald, X.: Intracranial subdural hematoma during spinal anesthesia. Med Trop (Mars). 1998; 58: 271-272.

46. Powell, D.F., Baker, H.L. Jr., Laws, E.R. Jr.: The primary angiographic findings in pituitary adenomas. Radiology. 1974 Mar; 110: 589-595.

47. Racadot, J., Gremain, J., Kujas, M., Drouet, Y., Olivier, L.: Involvement of arterial vessels in the blood supply to adenomas of the human pituitary, functional implications. Bull Assoc Anat (Nancy). 1986 Sep; 70: 5-12.

48. Raymond, J.R., Raymond, P.A.: Post-lumbar puncture headache. Etiology and management. West J Med. 1988 May; 148: 551-554.

49. Rovit, R.L., Fein, J.M.: Pituitary apoplexy: a review and reappraisal. J Neurosurg 1972; 37: 280-288.

50. Schechter, J., Goldsmith, P., Wilson, C., Weiner, R.: Morphological evidence for the presence of arteries in human prolactinomas. J Clin Endocrinol Metab 1988; 67: 713-719.

51. Semple, P.L., Jane, J.A., Laws, E.R.: Clinical relevance of precipitating factors in pituitary apoplexy. Neurosurgery 2007; 61: 956-962.

52. Settipani, N., Piccoli, T., La Bella, V., Piccoli, F.: Cerebral venous sinus expansion in post-lumbar puncture headache. Funct Neurol 2004; 19: 51-52.

53. Sheehan, H.L., Stanfield, J.P.: The pathogenesis of post-partum necrosis of the anterior lobe of the pituitary gland. Acta Endocrinol 1961; 37: 479-510.

54. Shigeki, M., Tsutomu, E.: Spontaneous Intracranial Hypotension Complicating Subdural Hematoma: Unilateral Oculomotor Nerve Palsy Caused by Epidural Blood Patch. Neurol Surg 2001; 29: 747-753.

55. Shimazu, N., Oba, H., Aoki, S., Monzawa, S., Makita, K., Araki, T.: Pituitary enlargement in spontaneous intracranial hypotension on MRI. Nippon Igaku Hoshasen Gakkai Zasshi. 1998 Jun; 58: 349-352.

56. Slowinski, J., Szydlik, W., Sanetra, A., Kaminska, I., Mrowka, R.: Bilateral chronic subdural hematomas with neurologic symptoms complicating spinal anesthesia. Reg Anesth Pain Med. 2003 Jul-Aug; 28: 347- 350.

57. Stanfield, J.P.: The blood supply of the human pituitary gland. J Anat. 1960 Apr; 94: 257-273.

58. Strandgaard, S., Andersen, G.S., Ahlgreen, P., Nielsen, P.E.: Visual disturbances and occipital brain infarct following acute, transient hypotension in hypertensive patients. Acta Med Scand 1984; 216: 417-422.

59. Strandgaard, S.: Autoregulation of cerebral blood flow in hypertensive patients. The modifying influence of prolonged antihypertensive treatment on the tolerance to acute, druginduced hypotension. Circulation. 1976 Apr; 53: 720-727.

60. Thomke, F., Mika-Gruttner, A., Visbeck, A., Bruhl, K.: The risk of abducens palsy after diagnostic lumbar puncture. Neurology. 2000; 54: 768-769.

61. Tien, R.D.: Sequence of enhancement of various portions of the pituitary gland on gadolinium-enhanced MR images: correlation with regional blood supply. AJR Am J Roentgenol. 1992 Mar; 158: 651-654.

62. Turner, H.E., Nagy, Z., Gatter, K.C., Esiri, M.M., Harris, A.L., Wass, J.A.: Angiogenesis in Pituitary Adenomas and the Normal Pituitary Gland. Clin Endocrinol Metab. 2000 Mar; 85: 1159-1162.

63. Verdú, M.T., Martínez-Lage, J.F., Alonso, B., Sánchez-Ortega, J.L., Garcia-Candel, A.: Non-surgical management of intracranial subdural hematoma complicating spinal. Neurocirugía 2007; 18: 40-43.

64. Verrees, M., Arafah, B.M., Selman, W.R.: Pituitary tumor apoplexy: characteristics, treatment, and outcomes. Neurosurg Focus. 2004 Apr 15; 16: E6.

65. Wakai, S., Yamakawa, K., Manaka, S., Takakura, K.: Spontaneous intracranial hemorrhage caused by brain tumor: its incidence and clinical significance. Neurosurgery. 1982 Apr; 10: 437-444.

66. Xuereb, G.P., Prichard, M.M., Daniel, P.M.: The arterial supply and venous drainage of the human hypophysis cerebri. Q J Exp Physiol Cogn Med Sci. 1954; 39: 199-217.

67. Yamauchi, T., Takano, H., Miyamoto, Y., Sawa, Y., Matsumiya, G., Matsuda, H.: Subdural hematoma induced by cerebrospinal fluid drainage: potentially catastrophic complication of spinal cord protection after thoracoabdominal aneurysm repair. Jpn J Thorac Cardiovasc Surg. 2004 Oct; 52:466-468.

68. Yatagai, T., Kusaka, I., Nakamura, T., Nagasaka, S., Honda, K., Ishibashi, S., Ishikawa, S.E.: Close association of severe hyponatremia with exaggerated release of arginine vasopressin in elderly subjects with secondary adrenal insufficiency. Eur J Endocrinol. 2003 Feb; 148: 221-226.

69. Yuh, W.T., Fisher, D.J., Nguyen, H.D., Tali, E.T., Gao, F., Simonson, T.M., Schlechte, J.A.: Sequential MR enhancement pattern in normal pituitary gland and in pituitary adenoma. AJNR Am J Neuroradiol. 1994 Jan; 15: 101-108.

70. Zayour, D.H., Selman, W.R., Arafah, B.M.: Extreme elevation of intrasellar pressure in patients with pituitary tumor apoplexy: relation to pituitary function. J Clin Endocrinol Metab. 2004 Nov; 89: 5649-5654.

71. Zeidan, A., Chaaban, M., Farhat, O., Baraka, A.: Cerebral rebleeding by spinal anesthesia in a patient with undiagnosed chronic subdural hematoma. Anesthesiology. 2006 Mar; 104: 613-614.

72. Zeidan, A., Farhat, O., Maaliki, H., Baraka, A.: Does postdural puncture headache left untreated lead to subdural hematoma? Case report and review of the literature. Int $\mathrm{J}$ Obstet Anesth 2006; 15: 50-58.

González-Tortosa, J.; Poza-Poza, M.; Ruiz-Espejo-Vilar, A.: Apoplejía de adenoma hipofisario no funcionante tras anestesia espinal. A propósito de dos casos. Neurocirugía 2009; 20: 484-493.

Correspondencia con los autores: tortosa@ctv.es 